\title{
Assurance-maladie et libéralisme économique: l'échec de l'initiative populaire du Parti socialiste suisse de 1974
}

Hadrien Buclin

\section{Résumé}

En 1974, le corps électoral suisse repousse lors d'une votation une initiative populaire du Parti socialiste suisse en faveur d'une réforme de l'assurance-maladie, ainsi qu'un contre-projet moins ambitieux élaboré par les autorités. Cet échec des partisans d'une réforme mérite d'être étudié, car il s'agit de la principale tentative, jusqu'à nos jours, de mettre en œuvre un modèle reposant sur un financement social et impliquant une couverture des risques étendue. De fait, dans les mois qui suivent le scrutin, la crise économique renforce les partisans d'une limitation du développement des prestations sociales et réduit du même coup la perspective de transformation en profondeur de l'assurance-maladie espérée par la gauche. L'échec socialiste de 1974 se solde ainsi par une consolidation du modèle libéral sur le plan économique, promu par la droite conservatrice et le patronat.

Mots-clés: assurance-maladie, Suisse, Parti socialiste, sécurité sociale, votation

\section{Summary}

In 1974, the Swiss citizens voted against a popular initiative aimed at reforming the health insurance, launched by the Swiss Socialist Party. They refused as well a less ambitious counter-proposal elaborated by the government. This failure of the left-wing reformers is worthy of interest. This was, indeed, the main attempt until now to implement a healthcare subsidization based on so-

Hadrien Buclin, Chercheur FNS senior et Archives sociales suisses, Faculté des Lettres, Section d'histoire, Quartier UNIL-Chamberonne, Bâtiment Anthropole, CH-1015 Lausanne,

Hadrien.Buclin@unil.ch 
cial funding that would provide a wide risk coverage. In fact, shortly after the vote, the emerging economic crisis rather reinforced the advocates of a limitation of social welfare benefits. This durably hindered the political Left's hopes of transforming in depth the Swiss healthcare system. The 1974 failure of the socialist initiative thus contributed to strengthen the conservative model, which received support from right-wing forces and the business community.

Keywords: health insurance, Switzerland, Socialist Party, social security, popular vote

En décembre 1974, le corps électoral refuse sans appel, par 70,2 \% des suffrages exprimés, une initiative populaire du Parti socialiste suisse (PSS) «pour une meilleure assurance-maladie». Le contre-projet élaboré par les autorités, qui propose une réforme de moindre ampleur, est lui aussi repoussé par $61,4 \%$ des voix. Malgré le maintien du statu quo, cette votation constitue un épisode significatif des affrontements sociopolitiques qui ont marqué l'histoire de l'assurance-maladie en Suisse. Il s'agit en outre de la principale tentative, jusqu'à nos jours, de mettre en œuvre un modèle reposant sur un financement social et impliquant une couverture des risques étendue.

De fait, dans les mois qui suivent ce scrutin, la crise économique, en interrompant près de trois décennies de croissance dynamique, renforce les partisans d'une limitation du développement des prestations sociales et réduit du même coup la perspective de transformation en profondeur de l'assurance-maladie espérée par la gauche. ${ }^{1}$ L'échec socialiste de 1974 se solde ainsi par une consolidation du modèle libéral sur le plan économique, promu par la droite gouvernementale. Ce modèle est fondé sur la concurrence entre les assureurs (les caisses maladie) et sur un financement assumé principalement par les assurés, caractérisé en outre par une faible solidarité entre bas et hauts revenus.

L'initiative socialiste de 1974 est certes souvent mentionnée dans les travaux de sciences sociales et politiques consacrés au développement de l'assurance-maladie. ${ }^{2}$ Cet épisode n'a cependant pas fait l'objet d'une étude historique, allant de sa genèse à son échec et aux conséquences de celui-ci, en passant par l'examen du processus parlementaire puis de la campagne de votation. ${ }^{3}$ C'est cette lacune que la présente contribution propose de combler,

1 Morabia 1983, 80; Moser 2008, 102; Rey 1983, 19-32; Ruoss 2018, 189-214; Studer 2012, 953-954.

2 Bridel 1990, 15-19; Moser 2008, 101-134; Sommer 1978, 457-532; Uhlmann/Braun 2011, $76-86$.

3 Ce constat n'a rien d'étonnant quand on sait que l'assurance-maladie, deuxième domaine le plus important de la protection sociale après celui des retraites, est pourtant l'un des moins bien investigués par la recherche historique, voir: Leimgruber 2011, 227-228. 
à l'aide de sources produites par les médias, l'administration fédérale, les organisations patronales ainsi que les partis et élus politiques. Cette documentation permet de mieux éclairer l'action des différents acteurs impliqués et ainsi de mieux comprendre les causes de l'échec socialiste. ${ }^{4}$

\section{La situation de l'assurance-maladie au tournant des années 1960-1970 et le lancement de l'initiative socialiste}

Au tournant des années 1960-1970, l'organisation de l'assurance-maladie en Suisse contraste, par ses principes de libéralisme économique, avec les modèles mis en place dans la plupart des pays d'Europe occidentale. ${ }^{5} \mathrm{Au}$ sortir de la Deuxième Guerre mondiale, à l'heure où les pays voisins développent des systèmes de sécurité sociale couvrant les risques de maladie, les partisans d'une telle voie en Suisse connaissent en revanche un revers cuisant, qui n'est pas sans présager celui de 1974. En 1949, le corps électoral helvétique refuse en effet par $75 \%$ des suffrages exprimés un complément à la loi fédérale sur la lutte contre la tuberculose.${ }^{6}$ Cette révision législative aurait impliqué une couverture maladie obligatoire pour les couches les plus modestes de la population, raison pour laquelle elle est combattue en référendum par des cercles patronaux, par la très conservatrice Ligue vaudoise et par des politiciens du Parti libéral, aiguillon traditionnel des partis bourgeois sur leur flanc droit. Ces milieux invoquent la tradition fédéraliste pour combattre le développement d'un État social centralisateur. Les sections nationales des trois partis de la droite gouvernementale, de leur côté, laissent la liberté de vote au moment du scrutin, en raison de leurs divisions internes.

Au-delà des arguments fédéralistes, l'issue de la votation de 1949, synonyme de fiasco pour les partisans du développement de l'État social, s'explique en particulier par la force électorale de l'alliance entre partis de la droite gouvernementale et organisations d'agriculteurs depuis le début du $\mathrm{XX}^{\mathrm{e}}$ siècle. ${ }^{7}$ Cette alliance, connue sous le nom de bloc bourgeois, relègue les gauches sociale-démocrate et communiste, principales forces engagées en faveur du développement de la sécurité sociale, dans une des positions les

4 J'aurais en outre voulu consulter les procès-verbaux de la direction du Concordat des caisses maladie suisses, afin de mieux comprendre l'action des assureurs dans le cadre de la votation de 1974. J'ai sollicité dans ce but l'association Santésuisse, qui a succédé au Concordat en 2002; il m'a toutefois été répondu que ces documents n'avaient pas été conservés.

5 Jens/Bernardi-Schenkluhn 1992, 179; Maarse/Paulus 2003, 585-614; Undritz 1988, 83; Leimgruber/Lengwiler/Togni 2013.

6 Lengwiler 2009, 182; Sommer 1978, 460.

7 Jost 2004, 691-693. 
plus minoritaires parmi les différentes configurations politiques nationales en Europe occidentale. ${ }^{8}$ Tous résultats cumulés, les partis de gauche, depuis l'introduction du suffrage proportionnel en 1919 jusqu'aux années 1970 (et même jusqu'à nos jours), ne dépassent pas le tiers des suffrages exprimés lors des élections nationales, alors que ces mêmes forces conquièrent des majorités électorales dans plusieurs pays d'Europe occidentale au sortir de la guerre. Les milieux conservateurs helvétiques favorables à une limitation du développement de l'État social profitent en outre du fait que la Suisse a été épargnée par les conséquences dramatiques du deuxième conflit mondial. Par contraste, dans un pays comme la Grande-Bretagne, la présence de dizaines de milliers de blessés de guerre incite le gouvernement à accélérer le développement d'une sécurité sociale publique et un accès gratuit aux soins médicaux, à plus forte raison vu la victoire de la gauche travailliste aux élections de 1945. ${ }^{9}$ Bien que le rapport Beveridge, qui sert de base au développement de la sécurité sociale britannique, soit débattu en Suisse, les représentants de la droite helvétique font valoir les différences majeures avec la situation de Grande-Bretagne pour justifier une voie beaucoup plus timorée que celle tracée par le célèbre économiste mandaté par le gouvernement de Winston Churchill. ${ }^{10}$

Le résultat de la votation de 1949 amène donc un premier coup d'arrêt au développement de la sécurité sociale d'après-guerre et consolide le modèle libéral-conservateur, fondé sur l'affiliation volontaire à une caisse maladie et sur le libre choix du catalogue de prestations couvertes, qui dépend du niveau de primes (ou cotisations) que l'assuré est prêt à assumer (excepté dans les cas où, pour une minorité de salariés du pays, une convention collective de travail prévoit une couverture maladie et en fixe les modalités). ${ }^{11}$

Cependant, dans les années qui suivent la votation de 1949, le développement des techniques médicales et du réseau hospitalier ainsi que l'amélioration des revenus du personnel soignant, entre autres, provoquent une augmentation soutenue des coûts de la santé. Ainsi, entre 1950 et 1975, la part des coûts de la santé dans un produit intérieur brut lui-même en forte croissance passe de 3,5 à $7 \% .^{12}$ Cette évolution, en poussant à la hausse les primes payées par les assurés, questionne la viabilité du système helvétique;

8 Degen 1993, 129.

9 Thorpe 2008, 112-116.

10 Monachon 2002, 321-329; Degen 2005, 33.

11 Lettre de l'Association suisse des invalides au Conseil fédéral, non-datée [vers avril 1967], Archives fédérales suisses (désormais AFS), J2.322-01, 2012/119, vol. 412.

12 Gilliand 1990a, 23; relevons que les travaux de Pierre Gilliand sont marqués par les lacunes des statistiques sociales en Suisse et que cet auteur a beaucoup œuvré pour les combler, voir: Gilliand/Rossini 1997, V. 
à plus forte raison compte tenu du développement des modèles de sécurité sociale dans les pays voisins, où les cotisations proportionnelles au revenu permettent de limiter l'impact négatif de la hausse des coûts de santé sur le pouvoir d'achat des milieux populaires.

En 1964, les autorités réagissent par une révision de la Loi sur l'assurance-maladie. Celle-ci, en raison de son caractère limité, ne suscite pas d'opposition référendaire, bien qu'elle ait fait l'objet d'âpres débats entre les acteurs économiques et politiques (notamment entre les assureurs et les médecins sur la question des tarifs médicaux).${ }^{13}$ Cette révision ouvre certes la voie à une augmentation des subsides de la Confédération aux caisses maladie, désormais indexés sur l'évolution des frais de santé par assuré. Il s'agit ainsi de limiter le poids croissant des primes dans le budget des ménages de condition modeste. La réforme précise aussi le catalogue de prestations minimales que les assureurs subventionnés doivent couvrir. ${ }^{14}$ Cependant, elle ne met pas en cause l'esprit libéral-conservateur du système, pour au moins trois raisons.

Premièrement, le caractère facultatif de l'assurance demeure au plan fédéral, bien que les cantons aient la possibilité de rendre l'affiliation obligatoire. Comme un nombre croissant de cantons choisissent cette option, le pourcentage de personnes assurées passent certes de $48 \%$ en 1945 à $90 \%$ de la population totale en $1971 .{ }^{15}$ Toutefois, l'absence d'affiliation obligatoire au plan fédéral continue à provoquer de fortes inégalités d'accès à l'assurance-maladie. Parmi la minorité de personnes non-assurées au début des années 1970, on trouve aussi bien des ménages trop pauvres pour payer une cotisation que des personnes fortunées, et n'ayant pour cette raison pas besoin d'une assurance: la solidarité entre les ménages les plus pauvres et les plus riches s'en trouve dès lors réduite. ${ }^{16}$ Parmi les personnes dégageant des revenus trop modestes pour s'assurer, les femmes sont surreprésentées, comme l'analyse le Groupe des femmes socialistes dans une lettre au conseiller fédéral en charge du dossier:

Les femmes salariées appartiennent aux couches les plus basses de salaires et cherchent à éviter de hautes charges de primes d'assurance en renonçant à s'assurer. Pour les femmes mariées et non salariées des couches économiquement faibles de la population, on «épargne», en premier lieu, les primes de la femme. On estime en effet que la femme au foyer peut mieux se ménager que le mari dans sa profession. ${ }^{17}$

13 Kocher 1972; Lengwiler/Rothenbühler 2004, 74-78.

14 Undritz 1988, 76.

15 Gilliand 1990b, 337.

16 Journal de Genève, «Les caisses refusent d'assurer les 'mauvais risques' seulement», 24 juin 1972.

17 Lettre du Groupe des femmes socialistes au conseiller fédéral Hans Peter Tschudi, 31 juillet 1972, Secrétariat central du PSS 1974, 173. 
Certaines caisses refusent par ailleurs l'adhésion des personnes de plus de 60 ans, considérées comme ayant de trop forts risques de maladie. D'autres assureurs demandent aux personnes de cette tranche d'âge désireuses de s'affilier une cotisation trois fois plus élevée que pour un individu de 30 ans. ${ }^{18}$ Les journaux bourgeois connus pour leur lectorat à fort pouvoir d'achat, à l'instar de la Neue Zürcher Zeitung (NZZ), diffusent nombre de publicités pour des assureurs privés, proposant des contrats à des prix élevés pour les personnes âgées. ${ }^{19}$

Même dans les cantons qui rendent obligatoire l'assurance pour les habitants de plus de 60 ans ayant des petits revenus, la situation sociale demeure problématique. Un élu au Parlement national pour le Parti suisse du travail (PST), une petite organisation prosoviétique, dénonce ainsi la «situation absurde du canton de Vaud par exemple, qui a rendu l'assurance-maladie et hospitalière obligatoire pour les personnes de plus de 60 ans dont le revenu n'atteint pas un certain montant et qui forment le groupe social représentant les plus gros risques». ${ }^{20}$ Le résultat en est, explique-t-il, que ces personnes doivent payer de très lourdes cotisations, sans aucune garantie d'indemnité journalière en cas de maladie. Le député de gauche rappelle aussi qu'une personne qui contracte une assurance alors qu'elle souffre déjà d'une maladie chronique peut se voir refuser tout remboursement durant les cinq premières années suivant son affiliation à la caisse. ${ }^{21}$ Quant au calcul des primes en fonction du risque, il ne pénalise pas seulement les personnes âgées, mais aussi les femmes, qui paient des cotisations supérieures de $10 \%$ à celles des hommes, en raison de la couverture des frais de maternité. Cette discrimination est en particulier dénoncée par les dirigeants socialistes au moment du lancement de l'initiative «pour une meilleure assurance-maladie».22

Outre l'absence d'affiliation obligatoire au niveau fédéral, un deuxième aspect du système révèle son caractère limité sous l'angle de la sécurité sociale: les frais de santé remboursés en sus d'une liste de prestations minimales dépendent du montant de la prime choisi par l'assuré. Cas presque unique en Europe occidentale, les soins dentaires ne sont pas couverts par le catalogue de base. ${ }^{23}$ Fait plus problématique encore pour les assurés de

18 Conseil fédéral 1973, 928; La Brèche, «Assurance-maladie et accident: la réforme», 27 août 1974.

19 P. ex.: NZZ, «Krankenversicherung für über 60jährige», 10 avril 1974.

20 Armand Forel, séance du Conseil national du 26 novembre 1973, Bulletin officiel de l'Assemblée fédérale, 1437.

21 Ibid; voir aussi: Despland 1990, 48.

22 Alexandre Berenstein, procès-verbal (désormais PV) du congrès extraordinaire du PSS du 2 novembre 1969, Secrétariat central du PSS 1969, 43.

23 Delessert/Barras 2011, 102-104. 
condition modeste, les remboursements s'avèrent limités en matière d'hospitalisations de longue durée. En effet, si les caisses remboursent les soins à l'hôpital, elles ne couvrent généralement pas les frais de pension hospitalière. ${ }^{24}$ L'indemnité pour perte de gain en cas de maladie prévue par les caisses pour les 360 premiers jours (passés lesquels l'assurance-invalidité, introduite en 1960, prend le relai) est donc généralement absorbée pour payer les frais d'hospitalisation. L'argent manque dès lors pour subvenir aux besoins de la famille d'un salarié hospitalisé. Comme le dénonce l'Association suisse des invalides dans une lettre au Conseil fédéral qui, dans la deuxième moitié des années 1960, plaide pour une nouvelle réforme rapide de l'assurance-maladie, les rapports cantonaux d'assistance montrent que la plupart des cas de pauvreté proviennent de la perte de gain du «chef de la famille» durant les 360 premiers jours. ${ }^{25}$ Les responsables de l'association estiment que l'introduction d'une assurance-maladie, couplée à une augmentation des rentes de vieillesse et d'invalidité, permettrait d'éradiquer ces cas de pauvreté. Du point de vue financier, cela supposerait, selon les calculs réalisés par cette association, d'introduire une cotisation à $9 \%$ du salaire. Or, ajoutent-ils:

avec une cotisation totale de $9 \%$, la Suisse serait encore toujours, en rapport avec les États européens progressistes, à la limite inférieure des cotisations versées à l'assurance-sociale. On connaît aujourd'hui des États scandinaves qui déduisent le double, c'est-à-dire dans lesquels $18 \%$ du salaire sont versés à l'assurance sociale. ${ }^{26}$

Enfin, troisième aspect témoignant de l'esprit bourgeois-conservateur du système, les primes ne tiennent pas compte du revenu. La loi fédérale de 1964 interdit aux cantons d'introduire un financement par une cotisation proportionnelle et liée au salaire, selon le modèle évoqué ci-dessus par les responsables de l'Association suisse des invalides. Quant aux subsides publics, ils ne permettent de soulager financièrement les ménages que de manière limitée, $74 \%$ des frais de santé restant à leur charge en $1974 .{ }^{27}$ Les familles nombreuses sont en particulier pénalisées par le système, car elles voient s'additionner les primes pour chaque membre du ménage. ${ }^{28}$ Enfin, la révision de 1964 introduit une franchise de 20 à 30 francs, soit une participation mini-

24 Journal de Genève, «Votations du 8 décembre: l'assurance-maladie», 2 décembre 1974.

25 Lettre de l'Association suisse des invalides au Conseil fédéral, non-datée [vers avril 1967], AFS, J2.322-01, 2012/119, vol. 412. Rappelons par ailleurs, à propos de la prise de position de cette association, que dans le cadre de la mise en œuvre de l'assurance-invalidité en 1960, les partis bourgeois se sont opposés au remboursement par cette assurance des soins médicaux et des dépenses pharmaceutiques, voir Fracheboud 2015, p. 181.

26 Lettre de l'Association suisse des invalides au Conseil fédéral, non-datée [vers avril 1967], AFS, J2.322-01, 2012/119, vol. 412.

27 Leuthy 1973, 36.

28 NZZ, «Die Neuordnung der Krankenversicherung», 20 novembre 1974. 
male directe de l'assuré aux frais, présentée par les milieux bourgeois comme permettant de réduire le recours abusif aux consultations médicales. ${ }^{29}$

C'est pour répondre à ces problématiques que la direction du PSS considère l'amélioration de l'assurance-maladie comme un chantier prioritaire. En collaboration avec son allié historique, l'Union syndicale suisse, qui compte alors quelque 450000 salariés affiliés, la direction du PSS élabore à la fin des années 1960 une initiative populaire sur cette thématique. Pour les dirigeants sociaux-démocrates, l'initiative s'est en effet imposée dans les années d'après-guerre comme un moyen de pression sur les partis bourgeois, afin d'obtenir une extension progressive de la protection sociale. Ainsi, c'est en réaction au lancement de deux initiatives, l'une portée par le PST, l'autre par le PSS, que les autorités mettent en œuvre une assurance-invalidité en $1960 . .^{30}$

Dans un premier temps, le projet prévoit une extension de la sécurité sociale aussi bien dans le domaine de la maladie qu'en matière de retraites. Cette orientation ambitieuse est en particulier portée par l'aile gauche du parti, qui connaît une progression dans le sillage des mouvements sociaux de la fin des années 1960. Mais après quelques mois de réflexion, la direction du PSS décide de scinder le projet à travers le lancement de deux initiatives, en raison de la complexité de la matière et pour réduire le risque d'un cumul des oppositions à ses propositions. ${ }^{31}$ En matière de retraites, la direction du PSS se heurte de plus à la méfiance des directions syndicales. Celles-ci redoutent une trop forte extension de l'assurance publique aux dépens des caisses de pension d'entreprise, dont la gestion s'avère financièrement intéressante pour les organisations de salariés, tout en leur garantissant une certaine influence dans les relations de travail. ${ }^{32}$

L'initiative populaire pour une «meilleure assurance-maladie» - qui réunit 88527 signatures en quatre mois (alors que seules 50000 sont nécessaires à son aboutissement) - est déposée le 31 mai 1970. Le texte vise à ancrer dans la Constitution suisse quatre principes. ${ }^{33}$ Premièrement, il prévoit une obligation d'assurance pour les frais de maladie, d'accident, de soins dentaires et de maternité, ainsi qu'une couverture pour la perte de gain en cas de maladie ou accident de longue durée, y compris pour les travailleurs in-

29 Morabia 1983, 77.

30 Fracheboud 2015, 75-94; sur l'emploi de l'initiative populaire dans l'après-guerre comme moyen de pression en faveur de l'extension des prestations sociales, voir: Degen 2005, 35; Eichenberger/Leimgruber 2019, 97.

31 PV du congrès extraordinaire du PSS, 2 novembre 1969, Secrétariat central du PSS 1969, 42; voir aussi: Leimgruber 2008, 246.

32 Ibid.

33 Initiative fédérale «pour une meilleure assurance-maladie», en ligne: https://www.bk.ad$\mathrm{min.ch/ch/f/pore/vi/vis97t.html.}$ 
dépendants et les personnes sans activité lucrative (au début des années 1970, seuls les salariés du secteur industriel sont couverts par une assurance-accident obligatoire). Deuxièmement, il est question d'interdire toute franchise pour les maladies ou accidents de longue durée, les hospitalisations, opérations et accouchements. Troisièmement, les socialistes et leurs alliés demandent un financement reposant sur une cotisation prélevée en pourcent du salaire et assumée pour moitié par l'employeur. Une telle cotisation est déjà en vigueur pour l'Assurance-vieillesse et survivants (AVS), introduite en 1948, et pour l'Assurance-invalidité (AI), mise en œuvre en 1960. Ce mode de financement au caractère redistributif est aussi répandu dans les pays voisins. ${ }^{34}$ Enfin, l'initiative socialiste vise à encourager le développement par la Confédération et les assureurs d'une politique de prévention en matière de santé publique et d'accident.

Bien qu'elle impliquerait un tournant majeur de la politique sociale en matière de santé, relevons toutefois que l'initiative ne propose pas un service de santé nationalisé sur le modèle britannique ou scandinave. Afin de ménager les intérêts des caisses maladie, l'initiative ne prévoit pas non plus une caisse publique unique, mais demande de «tenir compte des caisses existantes» au moment de la mise en œuvre du projet par le Parlement, qui ferait suite à l'éventuelle acceptation du texte par le corps électoral. Pour ces raisons, les militants des organisations politiques situées à la gauche du Parti socialiste - électoralement faibles mais néanmoins très actives dans les années qui suivent mai 1968 - critiquent le caractère à leurs yeux timoré du projet. ${ }^{35}$

\section{La stratégie des milieux bourgeois face à l'initiative du PSS}

Le texte socialiste suscite une large opposition, qui se manifeste au sein des trois partis de la droite gouvernementale, des associations patronales, des représentants des caisses maladie et de la Fédération des médecins suisses. Dans ces milieux, on fait en particulier valoir que le remboursement généralisé des médicaments et des soins conduirait, en déresponsabilisant la population, à une surconsommation médicale qui accélèrerait encore davantage l'augmentation des coûts de santé. ${ }^{36}$ Parmi les trois forces politiques de la

34 Conseil fédéral 1973, 927. L'aspect redistributeur et progressiste de ce modèle a par ailleurs été analysé par des économistes, à l'instar de: Friot 1998.

35 La Brèche, «La réforme de l'assurance-maladie», 7 octobre 1974 (La Brèche est l'organe de la Ligue marxiste révolutionnaire qui compte alors environ 400 membres en Suisse, voir: Challand 2000).

36 Conseil fédéral 1973, 929. 
droite gouvernementale, même le Parti démocrate-chrétien (PDC) - qui comprend pourtant une aile gauche liée à la Fédération suisse des syndicats chrétiens - repousse l'initiative lors de son congrès de 1974, par 151 voix contre $17 .{ }^{37}$ Les responsables de la Fédération des médecins suisses sont eux aussi hostiles à l'initiative socialiste. Les médecins libéraux craignent en particulier un contrôle public sur les tarifs, qui mettrait en cause la possibilité acquise à travers la révision de 1964 de prévoir une facturation plus élevée pour les hauts revenus, en échange de prestations étoffées. ${ }^{38}$

La hausse continue des primes-maladie fait pourtant craindre dans les milieux bourgeois que l'initiative socialiste puisse l'emporter en votation populaire. Certains représentants de la droite gouvernementale plaident donc pour un contre-projet qui serait soumis au vote en même temps que le texte socialiste..$^{39} \mathrm{~A}$ cette date, le recours à un contre-projet est en effet une option d'autant plus efficace pour combattre une initiative populaire que les citoyens favorables à une réforme n'ont pas la possibilité d'approuver les deux objets en même temps. Il faut en effet attendre la révision des droits populaires de 1987 et l'introduction du principe du double oui, avec question subsidiaire permettant d'indiquer une préférence entre deux objets, pour qu'un contre-projet ne s'oppose plus frontalement à une initiative populaire. ${ }^{40}$ Avant 1987, le lancement d'un contre-projet contribue donc à diviser le camp des partisans d'une réforme en éparpillant les suffrages.

Le contenu du contre-projet est cependant loin de faire l'unanimité parmi les opposants au texte socialiste. Ces dissensions inquiètent le conseiller fédéral Nello Celio qui, lors d'une séance du comité directeur du Parti radical-démocratique (PRD), principale force de la droite gouvernementale, met en garde le camp bourgeois: «La totale dispersion des points de vue amène avec elle le danger que l'initiative du PSS soit acceptée par le peuple. Il s'agit donc absolument de se soucier qu'un contre-projet substantiel et soutenable soit trouvé sous forme d'article constitutionnel, pour ne pas se faire avoir.» ${ }^{41}$ Dans cette optique, le Conseil fédéral soumet en 1973 un contre-projet constitutionnel qui prévoit une assurance obligatoire pour ce qu'on appelle alors les gros risques, soit les hospitalisations (44 \% des coûts de la santé au début des années 1970), ainsi qu'une indemnité journalière

37 Journal de Genève, «Les démocrates-chrétiens rejettent l'initiative socialiste sur l'assurance-maladie», 11 novembre 1974.

38 Journal de Genève, «Les médecins suisses diront non», 3 mai 1974.

39 Voir p. ex.: procès-verbal (désormais PV) de la séance du comité directeur du Parti radical-démocratique (PRD), 7 octobre 1970, AFS, J2.322-01, 2009/263, vol. 17.

40 Hottelier 2003, 666.

41 PV de la séance du comité directeur du PRD, 3 octobre 1972, AFS, J2.322-01, 2009/263, vol. 17 (ma traduction de l'allemand, désormais signalée par m. t.) 
pour les maladies de longue durée..$^{42}$ Les traitements ambulatoires resteraient en revanche couverts de manière facultative.

Mais la proposition gouvernementale suscite des critiques parmi les opposants à l'initiative socialiste, en particulier en provenance de deux milieux. Premièrement, les représentants des caisses dénoncent la scission de l'assurance en une couverture hospitalière obligatoire et une assurance ambulatoire facultative. ${ }^{43}$ Dès avant la proposition élaborée par le Conseil fédéral, le Concordat des caisses maladies a en effet déposé une pétition, munie de près de 600000 signatures, en faveur d'une obligation d'assurance généralisée, soit étendue au-delà des seuls gros risques. Ce modèle n'a toutefois pas été retenu dans le contre-projet du gouvernement. ${ }^{44}$ Le plaidoyer des représentants des caisses en faveur d'une telle variante s'explique par leur espoir d'amener la Confédération à augmenter les subsides aux assurances maladie, tout en instaurant une meilleure répartition des risques au sein de la population. Cela permettrait de réduire les difficultés financières des assureurs. Ceux-ci craignent en outre - quoique le Conseil fédéral s'en défende - que la couverture obligatoire pour les hospitalisations soit assumée par un organisme public centralisé, qui réduirait leur propre rôle au sein du système de santé. ${ }^{55}$ Une telle option est du reste combattue avec détermination et succès par les assureurs privés depuis l'entre-deux-guerres. ${ }^{46}$ Ils sont donc résolus, une fois encore, à ne pas baisser la garde.

La seconde opposition au contre-projet du gouvernement se focalise sur le mode de financement de l'assurance obligatoire pour les hospitalisations. Le Conseil fédéral a en effet repris la proposition socialiste, à savoir une cotisation sur les salaires assumée pour moitié par les employeurs. Le gouvernement propose cependant de la limiter à la couverture des hospitalisations et à l'indemnité journalière en cas de maladie de longue durée. Une telle proposition de financement est portée en particulier par le chef du Département de l'intérieur, le socialiste Hans Peter Tschudi, très actif sur les différents dossiers liés à l'extension des assurances sociales. Mais la proposition suscite l'ire de l'influente Union suisse du commerce et de l'industrie (USCI, dite aussi Vorort, du nom de sa direction) et de l'Union suisse des arts et mé-

42 Conseil fédéral 1973, 909.

43 Journal de Genève, «Le Concordat des caisses maladie de Suisse critique le principe de l'assurance gros risques», 6 juin 1973.

44 Journal de Genève, «Projet de révision de la LAMA: 600000 signatures déposées», 16 septembre 1972.

45 Gazette de Lausanne, «Le Concordat des caisses maladie suisses et la révision de l'assurance-maladie», 30 avril 1973; Conseil fédéral 1973, 939.

46 Lengwiler 2009, 170. 
tiers, associations défendant les intérêts, respectivement, des grands et petits patronats. ${ }^{47}$

Pour défendre leurs intérêts, ces associations peuvent compter sur des relais efficaces au sein des commissions du Parlement chargées d'examiner le contre-projet. Ce faisant, elles s'appuient sur une tactique formulée en interne par le secrétaire du Vorort en 1963: «Notre influence à Berne est plus forte lorsque nous restons dans l'ombre et que l'on ne parle pas trop de nous. ${ }^{48}$ Le patronat concentre ses efforts sur le Conseil des États. Des deux chambres du Parlement, il s'agit en effet de celle où le rapport de forces est le plus favorable à la droite, en raison de la surreprésentation des petits cantons conservateurs en son sein. Le Vorort y promeut avec succès un amendement limitant à $2 \%$ du salaire la ponction pour l'assurance hospitalière..$^{49}$ Par comparaison, l'initiative du Parti socialiste impliquerait une cotisation d'environ $6 \%$ non-plafonnée, c'est-à-dire susceptible d'augmenter en fonction de l'évolution des coûts de santé.

Lors de leur séance de direction, les dirigeants du Vorort se félicitent d'un tel résultat. Ils soulignent que si une telle variante passe la rampe du vote populaire, ce serait la première fois dans l'histoire du développement des assurances sociales helvétiques que le patronat obtiendrait l'inscription dans la Constitution du plafonnement d'une cotisation salariale. ${ }^{50}$ Lors du débat parlementaire, le conseiller fédéral Tschudi met en garde contre un tel plafonnement qui reviendrait, explique-t-il, à ce que les employeurs ne prennent à moyen terme aucune nouvelle part à la hausse des coûts des hospitalisations, celle-ci se répercutant uniquement sur les assurés et les budgets publics. ${ }^{51}$ Le chef du Département de l'intérieur juge de plus incongru l'introduction d'un pourcentage précis dans la Constitution.

Pour le patronat, la partie s'avère un peu plus compliquée dans la deuxième chambre du Parlement, le Conseil national, où les arguments de Tschudi sont davantage écoutés. Au PDC, en particulier dans les rangs des représentants de l'aile chrétienne-sociale, on juge excessif de plafonner les cotisations à un niveau aussi faible. ${ }^{52}$ Suite à une séance de conciliation entre

$47 \mathrm{PV}$ du comité directeur de l'Union suisse du commerce et de l'industrie (désormais USCI), 7 mai 1973, fonds Schweizerischer Handels- und Industrie-Verein, Archiv für Zeitgeschichte (Zurich; désormais AfZ).

48 Cité par Eichenberger 2016, 13.

49 PV du comité directeur de l'USCI, 27 août 1973, fonds Schweizerischer Handels- und Industrie-Verein, AfZ.

50 PV du comité directeur de l'USCI, 27 mai 1974, idem.

51 Séance du Conseil des États, 25 septembre 1973, Bulletin officiel de l'Assemblée fédérale, 541.

$52 \mathrm{NZZ,} \mathrm{«Fraktionsberatungen} \mathrm{vor} \mathrm{der} \mathrm{Märzsession} \mathrm{der} \mathrm{Bundesversammlung»,} 25$ février 1974. 
les deux Chambres, la cotisation est finalement plafonnée à $3 \%$. Pour rassurer les employeurs, la majorité parlementaire promet d'introduire un montant inférieur dans la loi de mise en œuvre, 3 \% étant un maximum qu'il ne s'agirait d'atteindre qu'à moyen terme..$^{53}$

Si les pressions du Vorort amènent, on le voit, des résultats tangibles, celles exercées par le Concordat des caisses maladie contre la scission de l'assurance peinent en revanche à se frayer un chemin parlementaire. Elles se heurtent en effet à l'hostilité des milieux bourgeois face à une assurance-obligatoire étendue au-delà des seuls gros risques. Le Concordat réagit donc en s'alliant avec la Fédération des médecins suisses. On peut dire, à propos d'une telle alliance conclue in extremis, que nécessité fait loi, car ces deux milieux ont connu dans les années précédentes des relations très tendues en raison de leurs difficultés à s'entendre sur la fixation des tarifs médicaux. «Moutons et chiens de berger, unissons-nous car le loup arrive!», commente ironiquement un politicien libéral à propos de cet accord..$^{54}$

Assureurs et médecins proposent d'abandonner tout caractère obligatoire pour l'assurance mais réclament en revanche davantage de subsides publics aux caisses. Conscients des réticences du patronat à accepter un prélèvement sur les salaires - les dirigeants du Vorort sont intervenus à plusieurs reprises auprès de la Fédération des médecins pour les dissuader de soutenir une telle option ${ }^{55}$ - ces milieux proposent un financement à travers une imposition plus forte des produits nocifs pour la santé, en premier lieu l'alcool et le tabac. Artisan du compromis entre assureurs et médecins, le conseiller d'État radical Bruno Hunziker, en charge du Département de la santé du canton d'Argovie, prend la défense du financement par une taxation des produits nocifs en expliquant que «dans nos hôpitaux, les alcooliques représentent environ $30 \%$ des malades hommes et le nombre de ceux-ci augmentent continuellement dans les cliniques psychiatriques». ${ }^{56}$

Mais ce mode de financement, non seulement impopulaire, se heurte en outre aux puissants lobbies des cigarettiers et vignerons: comme l'explique un parlementaire radical vaudois, l'introduction d'un impôt sur le vin susciterait dans son canton une levée de boucliers qui irait de la droite jusqu'aux communistes. ${ }^{57}$ L'imposition des produits nocifs à la santé est ainsi repous-

53 Séance du Conseil national, 4 mars 1974, Bulletin officiel de l'Assemblée fédérale, 215.

54 NZZ, «Dreimal Ja und einmal Nein zu den eidgenössischen Abstimmungsvorlagen», 18 novembre 1974 (m.t.)

55 PV du comité directeur de l'USCI, 7 mai et 27 août 1973, fonds Schweizerischer Handelsund Industrie-Verein, AfZ.

56 Bulletin des médecins suisses, «Révision de la LAMA», 30 mai 1973.

57 André Martin, séance du Conseil national, 28 novembre 1973, Bulletin officiel de l'Assemblée fédérale, 1479 . Les vignerons vaudois et valaisans ont déjà à plusieurs reprises fait obstacle aux projets d'un impôt fédéral sur le vin dans les années précédentes, notamment en 
sée par le Conseil national par 89 voix contre 66. Privée de sa principale source de financement, la proposition des caisses et des médecins fait long feu.

En résumé, le contre-projet qui sort des débats parlementaires fait moins de concessions à l'initiative socialiste que ne l'aurait souhaité le conseiller fédéral Tschudi. Suite à la mobilisation des milieux patronaux et libéraux, non seulement la cotisation sur salaire pour le financement de l'assurance hospitalière est plafonnée à $3 \%$, mais le contre-projet maintient en outre le principe des primes personnalisées, ainsi que la participation directe des assurés aux frais des traitements ambulatoires sous forme de franchise.

Lorsque le contre-projet initial du Conseil fédéral a été rendu public, les dirigeants socialistes avaient envisagé de s'y rallier en retirant leur initiative, pour éviter la dispersion des voix lors de la votation et le risque d'un double refus. Mais vu l'affaiblissement du contre-projet lors des débats parlementaires, les responsables socialistes décident de maintenir leur texte. ${ }^{58} \mathrm{Ce}$ choix est aussi motivé par le récent retrait, au terme d'un débat interne houleux, de l'initiative sur les rentes de vieillesse au profit d'un contre-projet. ${ }^{59}$ Les dirigeants du PSS craignent de décevoir leur base en retirant à nouveau un texte, peu après ce premier épisode. ${ }^{60}$

\section{La campagne électorale et l'échec socialiste}

Malgré l'orientation plus conservatrice du contre-projet au sortir des débats parlementaires, la droite n'est pas unanime à s'y rallier. Certes, le Vorort soutient officiellement le contre-projet revu par les Chambres, de même que la Fédération des médecins suisses et le Concordat des caisses maladie. Il en va de même du grand vieux parti de la droite gouvernementale, le PRD. Mais la cotisation sur les salaires, même plafonnée, et l'obligation d'assurance, même restreinte au domaine hospitalier, restent en travers de la gorge de nombreux représentants du camp bourgeois. Ceux-ci caressent l'espoir d'obtenir un projet plus favorable à leurs intérêts en cas de double refus en votation. Ainsi, l'assemblée du PRD arrête son mot d'ordre de vote favorable au

1951, voir: Longchamp 2014, 504. De même, certains milieux patronaux avaient plaidé en vain dans les années 1950 pour un financement de l'assurance-invalidité par un impôt sur l'alcool, voir Fracheboud 2015, 160-168.

58 Journal de Genève, «Le PSS maintient son initiative», 1er avril 1974.

59 PV du comité directeur du PSS, 16 février 1974, fonds PSS, Schweizerisches Sozialarchiv (Zurich; désormais Soz.), Ar. 1.110.63.

60 Journal de Genève, «L'USS propose au PSS de ne pas retirer son initiative», 26 mars 1974. 
contre-projet sur un score relativement serré: 89 voix contre $59 .{ }^{61}$ Plusieurs importantes sections cantonales du parti basculent dans le camp des opposants de droite au contre-projet, dont celles d'Argovie, Berne et Genève. Les caisses maladie s'y rallient aussi sans enthousiasme, les délégués du Concordat soutenant le contre-projet par 363 voix contre 198. ${ }^{62}$ De même, le comité de la Fédération des médecins suisses y apporte un soutien très tempéré, rappelant qu'une assurance obligatoire contre les gros risques suppose une «pression exercée par l'État sur les citoyens [... amenant] le risque d'entrâ̂ner des rapports impersonnels entre médecin et patient». ${ }^{63}$

Ces divisions au sein du camp bourgeois favorisent l'essor d'un comité pour le rejet du contre-projet. Celui-ci se montre de plus en plus actif dans les semaines précédant la votation, grâce à l'afflux de dons collectés notamment dans certains cercles d'employeurs mécontents du ralliement du Vorort à la variante issue du Parlement. Outre des radicaux en désaccord avec la ligne majoritaire de leur parti, ce comité compte également des représentants du Parti libéral, déjà fer de lance après la guerre du référendum contre la révision de la loi sur la tuberculose, ainsi que des médecins rejetant la position de leur fédération nationale. Enfin, une majorité de dentistes s'engagent pour le double non, car le contre-projet prévoit une couverture partielle des soins dentaires, qui leur fait craindre un contrôle public sur les tarifs. ${ }^{64}$

Le comité pour le double non fait valoir qu'une nouvelle ponction de $3 \%$ sur les salaires, qui s'ajouterait à celles prélevées pour l'AVS et l'AI, menacerait la survie économique de nombre de petites entreprises. La cause des petits patrons séduit aussi à l'extrême droite, l'Action nationale - qui rencontre alors un fort écho à travers ses initiatives populaires pour la réduction de l'immigration - se ralliant au mot d'ordre du double non. ${ }^{65}$ Le spectre d'une hausse excessive des cotisations salariales est pourtant contesté par la gauche, dont les représentants rappellent que la contribution des employeurs est significativement plus lourde dans les pays voisins, sans que cela n'entraîne de difficultés économiques majeures.

En dépit de leurs divisions face au contre-projet, les cercles bourgeois sont en tout cas unanimes à combattre l'initiative socialiste et n'hésitent pas à dramatiser les conséquences de celles-ci. Des enquêtes paraissent dans la

61 NZZ, «Dreimal Ja und einmal Nein zu den eidgenössischen Abstimmungsvorlagen», 18 novembre 1974.

62 Vaterland, «Krankenkasse gegen die SP-Initiative», 20 mai 1974.

63 Bulletin des médecins suisses, «Propositions fédérales sur l'assurance-maladie», 18 avril 1973.

64 Delessert/Barras 2011, 102-104.

65 NZZ, «Weitere Stellungnahmen zum 8. Dezember», 18 novembre 1974. 
presse conservatrice sur les défauts du système public britannique: sa gratuité générerait une surconsommation médicale de grande ampleur, des milliers de personnes possédant par exemple des lunettes sans les porter, certains les revendant même à l'étranger. ${ }^{66}$ Les éditorialistes de la $N Z Z$ expliquent que la proposition sociale-démocrate conduirait à une «fonctionnarisation» du corps médical. ${ }^{67}$ Certains médecins sont encore plus alarmistes, expliquant que le succès de la proposition du PSS représenterait le début d'un basculement de la Suisse vers un régime socialiste. ${ }^{68}$

Les médecins ne sont toutefois pas unanimes. Si la majorité d'entre eux soutient le contre-projet et qu'une minorité conservatrice appelle au double non, une autre minorité progressiste s'engage en revanche pour l'initiative. ${ }^{69}$ Il s'agit en particulier de jeunes praticiens hospitaliers, politisés à gauche à la faveur des mouvements de 1968. L'association des étudiants en médecine appelle ainsi au soutien de la proposition du PSS. ${ }^{70}$ Et en réaction à l'engagement de la Fédération des médecins suisses contre l'initiative, une minorité de gauche se structure au sein de celle-ci, qui plaidera dans les années suivantes pour que la Fédération adopte une ligne moins conservatrice. ${ }^{71}$

Du côté des opposants, le principal argument avancé contre l'initiative est, nous l'avons dit, celui de la déresponsabilisation de la population et de l'explosion des coûts de santé qu'une telle évolution provoquerait. Les représentants de la droite font ainsi valoir que l'initiative amènerait les personnes faisant l'effort d'une bonne hygiène dentaire à payer pour une minorité de négligents. ${ }^{72}$ Dans le même ordre d'idées, un membre du comité central de la Fédération des médecins suisses rappelle la tendance des patients à la surconsommation médicale:

Bien de ceux qui fréquentent les salles d'attente recherchent auprès du médecin ce que ce dernier, dans sa qualité spécifique de médecin, ne peut leur donner: une aide dans tous les problèmes imaginables, la solution de questions touchant au mode de vie quand ce n'est pas, tout simplement, la recherche d'un allié dans la lutte contre l'ennui. ${ }^{73}$

Face à l'argument de la surconsommation médicale qui entraînerait une explosion des coûts, une thèse simple et répétée de manière incessante durant la campagne, les responsables socialistes n'ont pas la partie facile, à l'image

66 P. ex.: Der Bund, «Licht und Schatten des britischen Gesundheitsdienstes», 23 mars 1973. 67 NZZ, «Der Arzt in der sozialen Krankenversicherung», 12 août 1974.

68 NZZ, «Stellungnahmen zur Revision der Krankenversicherung», 26 novembre 1974.

69 Bulletin des médecins suisses, «Weitere Aktionskomitees», 30 octobre 1974.

70 Journal de Genève, «Votation du 8 décembre: l'assurance-maladie», 2 décembre 1974.

71 Lengwiler/Rothenbühler 2004, 82-83.

72 Ibid.

73 J.[?] Imfeld, Bulletin des médecins suisses, «Révision de la LAMA», 2 mai 1973. 
de la parlementaire socialiste Gabrielle Nanchen, qui explique en réponse à la droite:

Si, pour se maintenir en santé, les assurés ressentent la nécessité de certaines prestations médicales et pharmaceutiques, qui peut juger du bien-fondé de ces besoins? Certains individus supportent les maux de tête et les insomnies stö̈quement, sans recourir à des médicaments. D'autres, pour pouvoir être efficaces à leur travail, ont besoin de prendre des aspirines et des somnifères. Les premiers font-ils de la sous-consommation médicale? Les autres font-ils de la surconsommation? ${ }^{74}$

Au-delà des échanges d'arguments, la disproportion de moyens entre les deux camps pour les diffuser dans la population est grande, bien que le PSS et l'USS aient mandaté un bureau de publicité (ce qui témoigne au passage d'une professionnalisation des campagnes politiques de gauche durant les années 1970). ${ }^{75}$ Selon l'estimation du secrétaire de l'USS Fritz Leuthy, les opposants à l'initiative investiraient 2,5 millions dans la campagne, contre 400000 francs pour ses partisans. ${ }^{76}$

Cette disproportion de moyens s'ajoute à d'autres facteurs structurels qui désavantagent la gauche helvétique sur le plan électoral et contribuent à expliquer la défaite socialiste. Outre la dispersion des voix entre contre-projet et initiative, déjà évoquée, il faut en particulier rappeler que les étrangers, qui constituent en 1974 environ $16 \%$ de la population, sont exclus des droits politiques. ${ }^{77}$ Or, ces derniers sont surreprésentés au sein des couches de salariés les plus modestes, soit parmi ceux qui ont le plus grand intérêt matériel à soutenir l'initiative. L'abstention élevée, de l'ordre de $60 \%$, pèse aussi négativement sur la participation de l'électorat populaire au scrutin.

Enfin, un facteur conjoncturel a probablement influencé le résultat de la votation. Il s'agit des premiers symptômes de la crise économique qui se manifestent dans les derniers mois de l'année $1974 .^{78} \mathrm{~A}$ en croire l'analyse de certains responsables socialistes au lendemain de la votation, ce contexte aurait renforcé les craintes de la population, entretenues par la droite, face à une extension des dépenses sociales qui ne serait pas supportable pour les entreprises. $^{79}$

Cela dit, l'ampleur de la défaite socialiste ne doit pas non plus être exagérée, dans la mesure où le cumul des voix de l'initiative (384000) et du contre-projet (458 000) donne une majorité aux partisans d'une révision de

74 Séance du Conseil national du 27 novembre 1973, Bulletin officiel de l'Assemblée fédérale, 1448.

75 PV du comité directeur du PSS, 17 janvier 1974, fonds PSS, Soz., Ar. 1.110.63.

76 PV du comité directeur du PSS, 30 mars et 14 décembre 1974, fonds PSS, idem.

77 Statistique historique de la Suisse HSSO, 2012. Tab. B.43, en ligne: www.hsso.ch/2012/b/4.

78 P. ex.: Journal de Genève, «La Maison Blanche annonce une récession aux États-Unis», 13 novembre 1974.

79 Pierre Aguet, «Des vents froids venus du nord», Tribune socialiste vaudoise, 30 janvier 1975. 
l'assurance maladie, d'environ 840000 voix contre 550000 pour le double non de droite. Les éditorialistes sociaux-démocrates ne manquent pas de le rappeler au lendemain du vote, pour relativiser l'ampleur de leur défaite..$^{80}$

Cependant, la dégradation de la situation économique dans les mois suivants anéantit les espoirs que peut alors susciter cette majorité de voix favorables à une avancée sociale dans le domaine de l'assurance-maladie. Les déficits provoqués dans les comptes de la Confédération par la récession (ainsi que par les allègements fiscaux consentis durant les années précédentes en faveur des contribuables aisés) $)^{81}$ amènent en effet les autorités à couper dans les subventions aux caisses maladie. Elles décident en outre dans la foulée d'augmenter la franchise. Les protestations non seulement de la gauche mais aussi de certaines sections du PDC qui considèrent ces mesures d'économies comme trop «antisociales» ne dissuadent pas les autorités de poursuivre dans cette voie..$^{82}$

En 1976, l'influent directeur de l'Administration fédérale des finances, dans une note au conseiller fédéral radical Georges-André Chevallaz, fixe les priorités pour la prochaine révision de l'assurance-maladie: il s'agit, explique-t-il, «d'en finir avec l'automatisme qui lie de manière rigide la contribution de la Confédération à l'augmentation des coûts de santé», afin de dégager de nouvelles économies dans les comptes publics. ${ }^{83}$ De fait, la part des subventions de la Confédération dans les dépenses des caisses diminue de $18,3 \%$ en 1970 à $10,7 \%$ en $1985 .{ }^{84}$

Cette évolution est en outre facilitée par les mutations à l'œuvre au sein des caisses maladie. Une partie importante d'entre elles ont été fondées, au $\mathrm{XIX}^{\mathrm{e}}$ siècle et dans la première moitié du $\mathrm{XX}^{\mathrm{e}}$, dans un but idéal (entraide religieuse, mutualisme, syndicalisme, etc.). Leurs responsables, à la tête de petites structures, s'engageaient donc souvent pour une politique de santé progressiste. Mais dans la seconde moitié du $\mathrm{XX}^{\mathrm{e}}$ siècle, de nombreux fusions et rachats favorisent l'émergence d'entreprises de grande taille. Ainsi, en 1960, si l'on dénombre encore 1088 caisses pour 4,4 millions d'assurés, elles ne seront plus que 550 en 1980 , puis 110 en 2000, pour 7,6 millions d'assurés. ${ }^{85}$ Ces nouveaux acteurs adoptent les principes de l'économie à but lucratif et les méthodes des assurances commerciales, leurs prises de position

80 Berner Tagwacht, «Klare Mehrheit für Verbesserungen», 12 décembre 1974.

81 Longchamp 2014, 819.

82 Lettre ouverte de Dominique Föllmi, président du PDC du canton de Genève, au Conseil fédéral, 16 septembre 1975, AFS, E 6801/1985-125, vol. 55.

83 Note de Rudolf Bieri à Chevallaz, 2 avril 1976, AFS, E 6801/1985-125, vol. 55 (m. t.)

84 Guex 1998, 96; pour l'évolution à plus long terme, voir aussi: Streckeisen 2013 et 2017.

85 Streckeisen 2013, 39; Eichenberger/Leimgruber 2019, 108. 
politiques s'éloignant dès lors des idéaux d'entraide pour se rapprocher de celles des organisations patronales. ${ }^{86}$

Du côté des organisations patronales justement - elles dont l'influence prépondérante sur les projets de réforme de l'assurance-maladie a été illustrée à plusieurs reprises dans les pages qui précèdent - on assiste aussi à un durcissement des positions en raison de la récession économique. ${ }^{87} \mathrm{Si}$, à la veille de la crise, elles se montrent prêtes à accepter, certes du bout des lèvres, une faible cotisation sur les salaires comme une des sources de financement de l'assurance-maladie, dans les années suivantes, elles excluent désormais catégoriquement une telle ponction. ${ }^{88}$ Après la défaite socialiste de 1949, celle de 1974, couplée à la récession, amène donc à un nouveau renforcement d'un système de santé dans lequel le principe de sécurité sociale est réduit à la portion congrue.

\section{Sources publiées et bibliographie ${ }^{89}$}

Bridel, Danielle, «Histoire de l'assurance-maladie suisse», in: Pierre Gilliand (éd.), Assurance-maladie. Quelle révision? (Lausanne 1990) 15-19

Challand, Benoît, La Ligue marxiste révolutionnaire en Suisse romande (Fribourg 2000)

Degen, Bernard, Sozialdemokratie: Gengenmacht? Opposition? Bundesratspartei? (Zurich 1993)

Conseil fédéral, Message du Conseil fédéral à l'Assemblée fédérale à l'appui d'un projet modifiant la Constitution fédérale dans le domaine de l'assurance-maladie, accidents et maternité et rapport sur l'initiative populaire pour une meilleure assurance-maladie (Berne 1973) 908-955

Degen, Bernard, «Entstehung und Entwicklung des schweizerischen Sozialstaates», Etudes et sources 31 (2005) 17-48

Delessert, Thierry/Vincent Barras, «Assurance maladie et remboursement des soins dentaires I: les débats des années 1960-1970», Revue mensuelle suisse d'odontostomatologie (2011) 102-104

Despland, Béatrice, «La pauvreté par l'assurance-maladie» in: Pierre Gilliand (éd.), Assurance-maladie. Quelle révision? (Lausanne 1990) 47-56

Eichenberger, Pierre, Mainmise sur l'État social. Mobilisation patronale et caisses de compensation en Suisse (1908-1960) (Neuchâtel 2016)

Eichenberger, Pierre/Matthieu Leimgruber, «Business Interests and the Development of the Public-Private Welfare Mix in Switzerland, 1880-1990», in: Dennie

86 Streckeisen 2017, 351. Cette évolution majeure des caisses maladie devrait faire l'objet de recherches historiques, pratiquement inexistantes à ce jour.

87 Eichenberger/Leimgruber 2019, 100-101.

88 Tribune de Genève, «Assemblée des délégués du Vorort à Zurich», 18 septembre 1976.

89 Les sources non-publiées et les articles de presse des années 1970 ne sont pas référencés dans cette section mais seulement dans les notes de bas de page. 
Oude Nijhuis (éd.), Business Interests and the Development of the Modern Welfare State (Abingdon 2019) 84-109

Fracheboud, Virginie, L'introduction de l'assurance-invalidité en Suisse (19441960). Tensions au cæur de l'État social (Lausanne 2015)

Friot, Bernard, Puissances du salariat. Emploi et protection sociale à la française (Paris 1998)

Gilliand, Pierre, «Santé publique: évolution des coûts, sources de financement et perspectives», Assurance-maladie. Quelle révision? (Lausanne 1990a) 21-34

Gilliand, Pierre, «Statistique de l'assurance-maladie», Assurance-maladie. Quelle révision? (Lausanne 1990b) 337-343

Gilliand, Pierre/Stéphane Rossini, La protection sociale en Suisse. Recettes et dépenses, 1948-1997. Comparaisons avec les pays de l'Union européenne (Lausanne 1997)

Guex, Sébastien, L'argent de l'État. Parcours des finances publiques au XX $X^{e}$ siècle (Lausanne 1998)

Hottelier, Michel, «Suisse: réforme des droits populaires de rang fédéral», Revue française de droit constitutionnel (2003) 657-670

Jens, Alber/Brigitte Bernardi-Schenkluhn, Westeuropäische Gesundheitssysteme im Vergleich: Bundesrepublik Deutschland, Schweiz, Frankreich, Italien, Grossbritannien (Francfort 1992)

Jost, Hans Ulrich, «Menace et repliement», in: Georges Andrey/Beatrix Mesmer et al. (éds), Nouvelle histoire de la Suisse et des Suisses (Lausanne 2004) 683-770

Kocher, Gerhard, Verbandseinfluss auf die Gesetzgebung: Ärzteverbindung, Krankenkassenverbände und die Teilrevision 1964 des Kranken- und Unfallversicherungsgesetzes (Berne 1972)

Leimgruber, Matthieu, Solidarity without the State? Business and the Shaping of the Swiss Welfare State, 1890-2000 (Cambridge 2008)

Leimgruber, Matthieu, «État fédéral, État social? L'historiographie de la protection sociale en Suisse», Traverse. Revue d'histoire (2011) 217-237

Leimgruber, Matthieu/Martin Lengwiler/Carola Togni, Histoire de la sécurité sociale en Suisse, site internet en ligne sur: https://www.histoiredelasecuritesociale.ch/ (Berne 2013)

Lengwiler, Martin/Verena Rothenbühler, Macht und Ohnmacht der Ärzteschaft: Geschichte der Zürcher Ärzteverbands im 20. Jahrhundert (Zurich 2004)

Lengwiler, Martin, «Das verpasste Jahrzehnt. Krankenversicherung und Gesundheitspolitik (1938-1949)», in: Matthieu Leimgruber/Martin Lengwiler (éd.), Umbruch an der «inneren Front». Krieg und Sozialpolitik in der Schweiz, 1938-1948 (Zurich 2009) 165-183

Leuthy, Fritz, «Réforme de l'assurance-maladie», Revue syndicale suisse (1973) 24 36

Longchamp, Olivier, La politique financière fédérale, 1945-1958 (Lausanne 2014)

Maarse, Hans/Aggie Paulus, «Has Solidarity Survived? A Comparative Analysis of the Effect of Social Health Insurance Reform in Four European Countries», Journal of Health Politics, Policy and Law (2003) 585-614

Monachon, Jean-Jacques, «Le plan Beveridge et les débats sur la sécurité sociale en Suisse entre 1942 et 1945», Société suisse d'histoire économique et sociale (2002) 321-329 
Morabia, Alfredo, Médecine et socialisme: politiques sanitaires en Suisse et dans les sociétés capitalistes avancées (Lausanne 1983)

Moser, Julia, Der schweizerische Wohlfahrtsstaat (Francfort 2008)

Rey, Jean-Noël, Trop d'État. Essai sur la mise en cause de l'État-protecteur en Suisse (Lausanne 1983)

Ruoss, Matthias, «Selbstsorge statt gesellschaftliche Solidarität. Die Neuverhandlung der sozialen Verantwortung in der 'Krise des Sozialstaats'», in: Regula Ludi/ Matthias Ruoss et al. (éds), Zwang zur Freiheit. Krise und Neoliberalismus in der Schweiz (Zurich 2018)

Secrétariat central du PSS, Ausserordentlicher Parteitag, Sonntag, den 2. November 1969, in Bern (Berne 1969)

Secrétariat central du PSS, Rapport de gestion 1972/1973 (Berne 1974)

Sommer, Jürg, Das Ringen um soziale Sicherheit in der Schweiz (Diessenhofen 1978)

Streckeisen, Peter, «Suisse. La santé publique comme laboratoire du néolibéralisme», Chronique internationale de l'IRES (2013) 35-44

Streckeisen, Peter, «35 Jahre Sparpolitik im Gesundheitswesen», Bulletin des médecins suisses (2017) 350-352

Studer, Brigitte, «Ökonomien der sozialen Sicherheit», in: Patrick Halbeisen/Margrit Müller et al. (éds), Wirtschaftsgeschichte der Schweiz im 20. Jahrhundert (Bâle 2012) 923-974

Thorpe, Andrew, A History of the British Labour Party (Basingstoke, 2008)

Uhlmann, Björn/Dietmar Braun (éds), Die schweizerische Krankenversicherungspolitik zwischen Veränderung und Stillstand (Coire 2011)

Undritz, Nils, Le système de santé en Suisse (Lausanne 1988) 